J. Austral. Math. Soc. 20 (Series B) (1978), 290-314

\title{
ON TENSOR OPERATORS AND CHARACTERISTIC IDENTITIES FOR SEMI-SIMPLE LIE ALGEBRAS
}

\author{
M. D. GOULD
}

(Received 1 December 1977)

(Revised 12 April 1978)

\begin{abstract}
Tensor identities for finite dimensional representations of arbitrary semisimple Lie algebras are derived and are applied to the construction of left-projection operators which project out the shift components of tensor operators from the left. The corresponding adjoint identities are also derived and are used for the construction of right-projection operators. It is also shown that, on a finite dimensional irreducible representation, these identities may be considerably reduced. Commutation relations between the shift tensors of a tensor operator are also computed in terms of the roots appearing in the tensor identities.
\end{abstract}

\section{Introduction}

The importance of tensor operators is apparent in several branches of physics and mathematics. Firstly there are the state labelling problems in which a semi-simple Lie algebra $K$ is contained in a larger Lie algebra $L$ and whose vector space complement in $L$ (denoted $K^{\perp}$ ) transforms as a tensor operator of $K$;

$$
\left[K, K^{\perp}\right] \subseteq K^{\perp}
$$

Secondly, we have the possibility of inducing representations of the Lie algebra $L$ from a given representation of the Lie algebra $K$ by considering the properties of the tensor operator $K^{\perp}$. Such a process may be regarded as being analogous to the theory of induced representations for Lie groups. Finally, we may consider certain infinite dimensional representations of the Lie algebra $L$ which may be reducible into finite dimensional representations of a semi-simple sub Lie algebra $K$. 
We shall be primarily concerned with tensor operators for an arbitrary semisimple Lie algebra $L$ and their shift properties when acting on finite dimensional irreducible representations of $L$. A theorem of Baird and Biedenharn [1] states that the number of linearly independent tensor operators transforming with an irreducible representation, $V$, is equal to the dimension of $V$. Using characteristic identity techniques we shall show how a tensor operator may be resolved into its distinct shift components by application of right- and left-projection operators. The problem of determining all tensor operators in a given irreducible representation of a semi-simple Lie algebra has been studied in recent years by a number of authors $[13,14]$. The problem was essentially solved for vector operators for the Lie algebras $g l(n, F), s o(n, F)$ and $s p(2 n, F)$ in the pioneering works of Green [6] and Bracken and Green [2] who showed how the characteristic identities may be applied to the construction of projection operators which project out (from the left) the shift components of an arbitrary vector operator. This work has recently been generalized by Hannabuss [9] and Okubo [15] to tensor operators for arbitrary semi-simple Lie algebras.

In this paper we shall generalize the work of Green by considering an appropriate matrix $A$ over a semi-simple Lie algebra $L$ and its adjoint $A$. The characteristic equations of these operators are derived and it is shown how they may be applied to the construction of projection operators which project out the shift components of tensor operators. The application of the $A$-identity (adjoint identity) for arbitrary semi-simple Lie algebras is new and is used for the construction of right-projection operators. As we shall see the right-projection operators are more useful since they are the ones which must be applied in practice.

In Section 4 we shall show that the polynomial identities satisfied by the matrices $A$ and $A$ will in fact reduce on a given irreducible representation. We shall also give a simple criterion for determining which representations will occur in the Clebsch-Gordan decomposition of a tensor product between two irreducible representations. Moreover, it will be shown that there is a 1-1 correspondence between the factors appearing in the reduced identity and the representations occurring in the Clebsch-Gordan series.

In the final section of this paper we consider the situation in which we have a tensor operator $T$ for a semi-simple Lie algebra $L$ where $L$ and $T$ generate a larger Lie algebra $K$. In this way, we admit a wide variety of Lie algebras. Although many properties of tensor operators have been studied, little is known about the commutation relations satisfied by the shift components of a tensor operator. We conclude by developing a technique for determining such commutation relations for tensor operators with commuting components which may also be applied in certain cases to tensor operators with non-commuting components.

Throughout this paper we shall consider tensor operators for arbitrary semisimple Lie algebras. However, extensions to the reductive Lie algebras are often trivial and we shall not hesitate to apply our results to such cases in examples. 


\section{Polynomial identities and tensor operators}

Let $L$ be a semi-simple Lie algebra over a field $F$ and let $H$ denote a fixed Cartan subalgebra of $L$ with dual space $H^{*}$. We denote the set of roots of $L$ relative to $H$ by $\Phi$ and the set of positive roots by $\Phi^{+}$. Finally, let $\left\{x_{1}, \ldots, x_{l}\right\}(l=\operatorname{dim} L)$, be a basis of $L$ and let $\left\{x^{1}, \ldots, x^{\}}\right\}$be its dual basis with respect to the Killing form of $L$.

We call a collection of operators $\left\{T_{i}\right\}$ indexed like components of vectors in a representation space $V$ of a finite dimensional representation $\pi$ of $L$ a tensor operator of $L$ if the components $T_{i}$ transform according to the rule

$$
\left[x, T_{i}\right]=\pi(x)_{i}^{j} T_{j}, \quad x \in L,
$$

where $\pi(x)$ denotes the matrix representing $x$ on the space $V$.

Dually we call a collection of components $\left\{T^{i}\right\}$ a contragredient tensor operator of $L$ if the components transform according to the rule

$$
\left[x, T^{i}\right]=-\pi(x)_{j}^{i} T^{j} .
$$

If $\pi$ is an irreducible representation of $L$ with highest weight $\lambda$ then we call the tensor operator an irreducible tensor operator of weight $\lambda$.

Following Kostant [11] we denote the universal enveloping algebra of $L$ by $U(L)$ and consider the map

$$
\partial: U(L) \rightarrow(\text { End } V) \otimes U(L)
$$

defined for $x \in L$ by

$$
\partial(x)=\pi(x) \otimes 1+1 \otimes x, \quad x \in L,
$$

which we extend to an algebra homomorphism to all of $U(L)$. In general $\partial(u)$ for arbitrary $u$ in $U(L)$ is a more complicated expression. For example, if $x, y \in L$ then

$$
\partial(x y)=\partial(x) \partial(y)=\pi(x y) \otimes 1+\pi(y) \otimes x+\pi(x) \otimes y+1 \otimes x y .
$$

When $z$ is an element of the centre $Z(L)$ of $U(L)$, Kostant shows that the operator $\partial(z)$ satisfies a certain polynomial identity. In this paper we shall consider the operator

$$
\tilde{z}=\frac{1}{2}(\partial(z)-\pi(z) \otimes 1-1 \otimes z) .
$$

Note that when acting on an irreducible representation $V(\mu)$ of $L$ with highest weight $\mu$ the operator $\partial(z)$ reduces to $\left(\pi \otimes \pi_{\mu}\right)(z)$ and $\tilde{z}$ reduces to the operator considered by Hannabuss [9] and others. 
Following Carey, Cant and O'Brien [3], when $z$ is the universal Casimir element $c_{L}$ we may express $\tilde{c}_{L}$ in terms of the basis elements of $L$ and their duals;

$$
\tilde{c}_{L}=\frac{1}{2} \sum_{r=1}^{l}\left(\pi\left(x_{r}\right) x^{r}+\pi\left(x^{r}\right) x_{r}\right)
$$

Throughout this paper we shall denote the operator $-\tilde{c}_{L}$ by $A$. We may regard $A$ as a matrix operator with entries

$$
A_{j}^{i}=-\frac{1}{2} \sum_{r=1}^{l}\left(\pi\left(x_{r}\right)_{j}^{i} x^{r}+\pi\left(x^{r}\right)_{j}^{i} x_{r}\right) .
$$

The matrix $A$ may be regarded as a generalization of the matrix $\tilde{a}$ appearing in the work of Bracken and Green $[2,6]$. We define an adjoint $A$ of the matrix $A$ by writing

$$
A_{j}^{i}=-A_{j}^{i}
$$

More generally we define

$$
\begin{aligned}
& \left(A^{m}\right)_{j}^{i}=A\left(A_{k}^{i m-1}\right)_{j}^{k}, \\
& \left(\bar{A}^{m}\right)_{j}^{i}=A_{j}^{k}\left(\bar{A}^{m-1}\right)_{k}^{i}
\end{aligned}
$$

(where the repeated affix $k$ is understood to be summed over). In this way we may define arbitrary polynomials in $A$ and $A$.

If $T$ is a contragredient tensor operator we may regard $A$ as an operator from the left on $T$ and $A$ as an operator from the right on $T$ by defining

$$
\begin{aligned}
& (A T)^{i}=A_{j}^{i} T^{j}, \\
& (T A)^{i}=T^{j} A_{j}^{i} .
\end{aligned}
$$

With this definition we have

$$
\begin{aligned}
(A T)^{i} & =A_{j}^{i} T^{j} \\
& =-\frac{1}{2} \sum_{r=1}^{l}\left(\pi\left(x_{\tau}\right)_{j}^{i} x^{r}+\pi\left(x^{r}\right)_{j}^{i} x_{\tau}\right) T^{j} .
\end{aligned}
$$

Substituting equation (2) into this expression gives

$$
\begin{aligned}
(A T)^{i} & =\frac{1}{2} \sum_{r=1}^{l}\left(\left[x^{r} x_{r}, T^{i}\right]-\left[x^{r}, T^{i}\right] x_{r}+\left[x_{r} x^{r}, T^{i}\right]-\left[x_{r}, T^{i}\right] x^{r}\right) \\
& =\left[c_{L}, T^{i}\right]+(T \bar{A})^{i} .
\end{aligned}
$$

Thus

$$
A T-T A=\left[c_{L}, T\right]
$$


On the other hand, we have

$$
\begin{aligned}
(T \bar{A})^{i}= & \frac{1}{2} T^{j} \sum_{r=1}^{l}\left(\pi\left(x_{r}\right)_{j}^{i} x^{r}+\pi\left(x^{r}\right)_{j}^{i} x_{r}\right) \\
= & \frac{1}{2} \sum_{r=1}^{l}\left(\pi\left(x_{r}\right)_{j}^{i} x^{r}+\pi\left(x^{r}\right)_{j}^{i} x_{r}\right) T^{j} \\
& +\frac{1}{2} \sum_{r=1}^{l}\left(\pi\left(x_{r}\right)_{k}^{i} \pi\left(x^{r}\right)_{j}^{k}+\pi\left(x^{r}\right)_{k}^{i} \pi\left(x_{r}\right)_{j}^{k}\right) T^{j} \\
= & \left(\pi\left(c_{L}\right)-A\right)_{j}^{i} T^{j} .
\end{aligned}
$$

Substituting this into equation (6) gives the result

$$
\left(2 A-\pi\left(c_{L}\right)\right) T=\left[c_{L}, T\right]
$$

Equations (6) and (7) are a generalization of equations (13), (24) and (64) appearing in the work of Green [6].

We shall now show that when $V$ is an irreducible module over $L$ that the matrices $A$ and $A$ satisfy a certain polynomial identity.

Throughout the remainder of this paper let $V(\lambda)$ denote a finite dimensional irreducible $L$ module with highest weight $\lambda$ and let $\pi_{\lambda}$ denote the representation of $L$ afforded by $V(\lambda)$. Henceforth we shall let $A$ denote the matrix

$$
A=-\frac{1}{2} \sum_{r}\left(\pi_{\lambda}\left(x_{r}\right) x^{r}+\pi_{\lambda}\left(x^{r}\right) x_{r}\right)
$$

From the Cartan decomposition of the semi-simple Lie algebra $L$ we may choose a basis for $L$ consisting of root space elements together with a basis for the Cartan subalgebra $H$. Hence let us consider a basis $\left\{h_{1}, \ldots, h_{m} ; x_{\alpha}, \alpha \in \Phi\right\}$ where $\left\{h_{1}, \ldots, h_{m}\right\}$ is a basis for $H$ and $x_{\alpha}$ is a non-zero element of the root space $L_{\alpha}$.

The dual basis may therefore be written

$$
\left\{h^{1}, \ldots, h^{m} ; x^{\alpha}, \alpha \in \Phi\right\}
$$

where $x^{\alpha}$ is the unique element of $L_{-\alpha}$ which is dual to $x_{\alpha}$ under the Killing form of $L$. We may therefore write the universal Casimir element in the form

$$
c_{L}=\sum_{i=1}^{m} h_{i} h^{i}+\sum_{\alpha \in \Phi} x_{\alpha} x^{\alpha} .
$$

Now let $V(\mu)$ be an irreducible finite dimensional representation of $L$ with highest weight $\mu$ and maximal weight vector $v_{0}$. Then

$$
c_{L} v_{0}=\sum_{i=1}^{m} \mu\left(h^{i}\right) \mu\left(h_{i}\right) v_{0}+\sum_{\alpha \in \Phi^{+}} \mu\left(t_{\alpha}\right) v_{0}
$$


where

$$
t_{\alpha}=\left[x_{\alpha}, x^{\alpha}\right]
$$

Since $c_{L}$ is an invariant of $L$ it must take the constant value shown on the entire space $V(\mu)$.

It is convenient to introduce the labelling operator $\Lambda$ which, when acting on an irreducible representation of $L$ with highest weight $\mu$, coincides with the weight $\mu$. We may regard $\Lambda$ as a "vector" operator with components $\Lambda\left(h_{i}\right)$ which take the constant values $\mu\left(h_{i}\right)$ on an irreducible representation of $L$ with the highest weight $\mu$.

We may therefore express the universal Casimir element in terms of the components of the labelling operator $\Lambda$ by writing

$$
c_{L}=\sum_{i=1}^{m} \Lambda\left(h^{i}\right) \Lambda\left(h_{i}\right)+\sum_{\alpha \in \Phi^{+}} \Lambda\left(t_{\alpha}\right)
$$

Suppose now that $\left\{\lambda_{1}, \ldots, \lambda_{k}\right\}$ are the distinct weights occurring in $V(\lambda)$. Then we may decompose an irreducible contragredient tensor operator $T$ with highest weight $\lambda$ into shift tensors $T_{[i}$, which decreases the eigenvalue of the labelling operator on an irreducible representation of $L$ by the weight $\lambda_{i}$;

$$
\Lambda\left(h_{j}\right) T_{[i]}=T_{[i]}\left(\Lambda-\lambda_{i}\right)\left(h_{j}\right)
$$

or

$$
\left[\Lambda\left(h_{j}\right), T_{[i]}\right]=-\lambda_{i}\left(h_{j}\right) T_{[i]} .
$$

Hence from equation (9) we obtain

$$
\left[c_{L}, T_{[i]}\right]=\left[-\sum_{j=1}^{m}\left(\lambda_{i}\left(h_{j}\right) \Lambda\left(h^{j}\right)+\Lambda\left(h_{j}\right) \lambda_{i}\left(h^{j}\right)+\lambda_{i}\left(h_{j}\right) \lambda_{i}\left(h^{j}\right)\right)-\sum_{\alpha \in \Phi^{+}} \lambda_{i}\left(t_{\alpha}\right)\right] T_{[i]^{*}}
$$

Substituting equation (11) into equation (7) gives the identity

$$
\left[A-\frac{1}{2} \pi_{\lambda}\left(c_{L}\right)+\frac{1}{2} \sum_{j=1}^{m}\left(\lambda_{i}\left(h_{j}\right) \Lambda\left(h^{j}\right)+\Lambda\left(h_{j}\right) \lambda_{i}\left(h^{j}\right)+\lambda_{i}\left(h_{j}\right) \lambda_{i}\left(h^{j}\right)\right)+\frac{1}{2} \sum_{\alpha \in \Phi^{+}} \lambda_{i}\left(t_{\alpha}\right)\right] T_{[i]}=0 .
$$

We may simplify this expression greatly by making the following substitutions (see Humphreys [10]);

$$
\begin{aligned}
\sum_{i=1}^{m} \Lambda\left(h_{i}\right) \Lambda\left(h^{i}\right) & =(\Lambda, \Lambda) \\
\sum_{\alpha \in \Phi^{+}} \Lambda\left(t_{\alpha}\right) & =(\Lambda, 2 \delta),
\end{aligned}
$$

where $\delta=\frac{1}{2} \sum_{\alpha \in \Phi_{+}} \alpha$ and (,) is the inner product on $H^{*}$ induced by the Killing form of $L$. We may interpret the operators $(\Lambda, \Lambda)$ and $(\Lambda, 2 \delta)$ as those operators which take the constant values $(\mu, \mu)$ and $(\mu, 2 \delta)$ respectively on an irreducible representation of $L$ with highest weight $\mu$. 
From this we obtain

$$
c_{L}=(\Lambda, \Lambda+2 \delta)
$$

and we may write equation (12) in the form

$$
\left(A-\frac{1}{2}(\lambda, \lambda+2 \delta)+\frac{1}{2}\left(\lambda_{i}, 2(\Lambda+\delta)+\lambda_{i}\right)\right) T_{[i]}=0 .
$$

Similarly substituting for $A$ using equations (6) and (7) gives us the equation

$$
T_{[i]}\left(\bar{A}-\frac{1}{2}(\lambda, \lambda+2 \delta)-\frac{1}{2}\left(\lambda_{i}, 2(\Lambda+\delta)-\lambda_{i}\right)\right)=0 .
$$

These equations may be regarded as a generalization of equations (14), (19) and (30) appearing in Green [6]. As in Green's case the matrices $A$ and $A$ satisfy the polynomial identities

$$
\begin{aligned}
& \prod_{i=1}^{k}\left(A-\frac{1}{2}(\lambda, \lambda+2 \delta)+\frac{1}{2}\left(\lambda_{i}, 2(\Lambda+\delta)+\lambda_{i}\right)\right)=0, \\
& \prod_{i=1}^{k}\left(\bar{A}-\frac{1}{2}(\lambda, \lambda+2 \delta)-\frac{1}{2}\left(\lambda_{i}, 2(\Lambda+\delta)-\lambda_{i}\right)\right)=0 .
\end{aligned}
$$

To see this suppose $V(\mu)$ is a finite dimensional irreducible representation of $L$ with highest weight $\mu \in \Lambda^{+}$. Then acting on $V(\mu)$ the operator $A$ becomes

$$
A=\frac{1}{2}\left(\pi_{\lambda} \otimes \pi_{\mu}\left(c_{L}\right)-\pi_{\lambda}\left(c_{L}\right) \otimes 1-1 \otimes \pi_{\mu}\left(c_{L}\right)\right) .
$$

Hence $A$ may be interpreted as an operator on the tensor product representation $V(\lambda) \otimes V(\mu)$.

Suppose now we write the Clebsch-Gordan decompositon of $V(\lambda) \otimes V(\mu)$ into irreducible representations of $L$ by

$$
V(\lambda) \otimes V(\mu)=\bigoplus_{i=1}^{k} n(i) V\left(\mu+\lambda_{i}\right),
$$

where $n(i)$ denotes the multiplicity of the weight $\lambda_{i}$ in $V(\lambda)$. Clearly this may be done due to the fact that there is a 1-1 correspondence between the weights occurring in the decomposition of $V(\lambda) \otimes V(\mu)$ and the weights $\lambda_{i}$ of $V(\lambda)$ (see, for example, Kostant [11]).

Then on each space $V\left(\mu+\lambda_{i}\right)$ the operator $A$ takes the constant value

$$
\frac{1}{2}\left[\chi_{\mu+\lambda_{j}}\left(c_{L}\right)-\chi_{\lambda}\left(c_{L}\right)-\chi_{\mu}\left(c_{L}\right)\right]
$$

which (using $\chi_{\nu}\left(c_{L}\right)=(\nu, \nu+2 \delta)$ for $\left.\nu \in H^{*}\right)$ equals

$$
\frac{1}{2}(\lambda, \lambda+2 \delta)-\frac{1}{2}\left(\lambda_{i}, 2(\mu+\delta)+\lambda_{i}\right)
$$


From this it is an easy matter to deduce that acting on the space $V(\mu)$ the operator $A$ satisfies the polynomial identity

$$
\prod_{i=1}^{k}\left(A-\frac{1}{2}(\lambda, \lambda+2 \delta)-\frac{1}{2}\left(\lambda_{i}, 2(\mu+\delta)+\lambda_{i}\right)\right)=0 .
$$

This result in fact follows from the easily established fact that a diagonal matrix $D$ with distinct eigenvalues $d_{1}, \ldots, d_{k}$ satisfies the polynomial identity

$$
\prod_{i=1}^{k}\left(D-d_{i}\right)=0 .
$$

A similar argument may be carried out for $A$.

Replacing the weight $\mu$ by our labelling operator $\Lambda$ the identities (14) and (15) are then seen to hold.

Note that these identities are independent of the basis chosen for $L$ and $V(\lambda)$.

Equations (14) and (15) are a generalization of the identities appearing in the work of Green. The identity satisfied by the matrix $-A$ is the identity appearing in the work of Hannabuss [9] and Carey, Cant and O'Brien [3].

From now on we write the identities (14) and (15) in the form

$$
\begin{aligned}
& \prod_{i=1}^{k}\left(A-\Lambda_{i}\right)=0 . \\
& \prod_{i=1}^{k}\left(\bar{A}-\bar{\Lambda}_{i}\right)=0,
\end{aligned}
$$

where

$$
\begin{aligned}
& \Lambda_{i}=\frac{1}{2}(\lambda, \lambda+2 \delta)-\frac{1}{2}\left(\lambda_{i}, 2(\Lambda+\delta)+\lambda_{i}\right), \\
& \bar{\Lambda}_{i}=\frac{1}{2}(\lambda, \lambda+2 \delta)+\frac{1}{2}\left(\lambda_{i}, 2(\Lambda+\delta)-\lambda_{i}\right) .
\end{aligned}
$$

Now let $\tilde{Z}$ denote the field $F\left(\Lambda_{1}, \ldots, \Lambda_{k}\right)$ where $F\left[x_{1}, \ldots, x_{k}\right]$ denotes the polynomial algebra over the underlying field $F$ in indeterminates $x_{1}, \ldots, x_{m}$ with field of quotients $F\left(x_{1}, \ldots, x_{m}\right)$.

Since the components $\Lambda\left(h_{i}\right)$ of the labelling operator $\Lambda$ take constant values on irreducible representations of $L$ we may regard $\Lambda$ as an invariant operator of $L$, that is,

$$
[\Lambda, L]=0 .
$$

From this we see that $\tilde{Z}$ is a field of invariants of $L$.

We have already shown in equation (13) that

$$
A T_{[i]}=\Lambda_{i} T_{[i]}
$$

By a simple induction argument we may show that if $p(x)$ is any polynomial over $\tilde{Z}$ then

$$
p(A) T_{[i]}=p\left(\Lambda_{i}\right) T_{[i]} .
$$


Substituting $p(x)=\Pi_{l \neq j}\left(x-\Lambda_{1}\right)$ into (18) gives

From this we obtain

$$
\prod_{l \neq j}\left(A-\Lambda_{\nu}\right) T_{[i]}=\prod_{l \neq j}\left(\Lambda_{i}-\Lambda_{l}\right) T_{[i]}
$$

$$
E_{j} T_{[i]}=\delta_{j i} T_{[i]}
$$

where $E_{j}$ are the projection operators

$$
E_{j}=\prod_{l \neq j}\left(\frac{A-\Lambda_{l}}{\Lambda_{j}-\Lambda_{l}}\right)
$$

We may write the decomposition of the contragredient tensor $T$ into its distinct shift components $T_{[i]}$ by writing

$$
T=\sum_{i=1}^{k} T_{[i] \cdot}
$$

If the weight $\lambda_{i}$ occurs with multiplicity $n(i)$ then $T_{[i]}$ may be further decomposed into $n(i)$ distinct parts each with the same shift property. Substituting equation (19) into equation (21) gives

$$
T_{(i)}=E_{i} T .
$$

Hence the operator $E_{i}$ projects out from the left, the shift components of the contragredient tensor $T$ corresponding to weight $\lambda_{i}$.

Using the $\bar{A}$-identity we may construct projection operators

$$
E_{i}=\prod_{i \neq i}\left(\frac{\bar{A}-\bar{\Lambda}_{l}}{\bar{\Lambda}_{i}-\bar{\Lambda}_{l}}\right)
$$

which satisfy

$$
T_{[i]}=T E_{i}
$$

and

$$
T_{[i]} E_{j}=\delta_{i j} T_{[i j}
$$

Hence the operators $E_{j}$ project out shift components of a contragredient tensor operator from the right.

The advantage of using the operators $E_{i}$ in this situation is that when acting on an irreducible representation of $L$ with highest weight $\mu$ the labelling operator $\Lambda$ may be replaced by the weight $\mu$ and the roots $\bar{\Lambda}_{i}$ appearing in the $A$ identity take known scalar values. Clearly this is not the case when we consider the left projections $E_{i}$.

Suppose now that $\tilde{T}$ is a tensor operator of highest weight $\lambda$. Then we may decompose $\tilde{T}$ into shift components $\tilde{T}_{[\mathfrak{l}\}}$,

$$
\tilde{T}=\sum_{i=1}^{k} \widetilde{T}_{[i]}
$$


where each shift component $\widetilde{T}_{[i]}$ satisfies

$$
\left[\Lambda(h), \tilde{T}_{[i]}\right]=\lambda_{i}(h) \tilde{T}_{[i]}, \quad h \in H .
$$

We may regard the matrix $A$ as an operator from the left on $\tilde{T}$ and the matrix $A$ as an operator from the right on $\tilde{T}$ by writing

and

$$
(A \tilde{T})_{\alpha}=A_{\alpha}^{\beta} \tilde{T}_{\beta}
$$

$$
(\tilde{T} A)_{\alpha}=\tilde{T}_{\beta} A_{\alpha}^{\beta} \text {. }
$$

By carrying out an analogous argument for tensor operators we may readily verify the identities

$$
\begin{gathered}
\tilde{T}_{[i]}\left(A-\Lambda_{i}\right)=0, \\
\left(\bar{A}-\bar{\Lambda}_{i}\right) \tilde{T}_{[i]}=0,
\end{gathered}
$$

where $\Lambda_{i}$ and $\bar{\Lambda}_{i}$ are the operators defined in (17).

From this it follows that

$$
\tilde{T}_{[i]}=E_{i} \tilde{T}=\tilde{T} E_{i} .
$$

Hence we see that the operators $E_{i}$ project out the shift components of tensors from the right while the $E_{i}$ project out the shift components from the left. In this situation it is more profitable to use the operators $E_{i}$.

From equations (14) and (15) we obtain

$$
A E_{i}=\Lambda_{i} E_{i} \text { and } A E_{i}=\bar{\Lambda}_{i} E_{i} \text {, }
$$

and more generally we may show by induction that if $p(x) \in \mathcal{Z}[x]$ then

$$
\begin{aligned}
& p(A) E_{i}=p\left(\Lambda_{i}\right) E_{i}, \\
& p(A) E_{i}=p\left(\Lambda_{i}\right) E_{i} .
\end{aligned}
$$

In particular $E_{i} E_{j}=\delta_{i j} E_{j}$ and $E_{i} E_{j}=\delta_{i j} E_{j}$ so that $E_{i}$ (respectively $E_{i}$ ) form an orthogonal set of idempotent operators. Moreover, from equations (21) and (22) we obtain a resolution of the identity on our space of tensor operators,

$$
\begin{aligned}
& 1=\sum_{i=1}^{k} E_{i}, \\
& 1=\sum_{i=1}^{k} E_{i} .
\end{aligned}
$$

Hence if $p(x)$ is a polynomial over $\mathcal{Z}$ then we may write

$$
\begin{aligned}
& p(A)=\sum_{i=1}^{k} p\left(\Lambda_{i}\right) E_{i}, \\
& p(A)=\sum_{i=1}^{k} p\left(\bar{\Lambda}_{i}\right) E_{i} .
\end{aligned}
$$


We may in fact extend this definition to arbitrary functions of the matrices $A$ and $A$. Following recent work of the author [5], we may define an inverse for the matrix $A$ by setting

$$
A^{-1}=\sum_{i=1}^{k} \Lambda_{i}^{-1} E_{i}
$$

Then, using the characteristic identity we see that

$$
\left(A^{-1}\right)_{j}^{i} A_{\bar{k}}^{j}=A_{j}^{i}\left(A^{-1}\right)_{k}^{j}=\delta_{k}^{i},
$$

and $A^{-1}$ satisfies the usual properties of an inverse matrix. Note that the inverse is only well defined on irreducible representations of the Lie algebra $L$ where the eigenvalues of the operators $\Lambda_{i}$ are all non-zero. Following the classical theory of numerical matrices we may define the determinant of the matrix $A$ to be the product of its eigenvalues $\Lambda_{i}$. In view of our previous remarks we see that the inverse of $A$ exists only on representations in which the determinant of $A$ is non-zero.

\section{Casimir invariants}

Following the notation of the previous section we may obtain a set of Casimir operators for a semi-simple Lie algebra by taking the trace of polynomials in $A$ and $A$ with respect to the $\lambda$-indices which occur. In particular we define the fundamental invariants

by

$$
I_{m}(\lambda)=\operatorname{tr}_{\lambda}\left(A^{m}\right)
$$

$$
I_{1}(\lambda)=-\frac{1}{2} \sum_{r=1}^{l}\left(\left[\operatorname{tr} \pi_{\lambda}\left(x_{r}\right)\right] x^{r}+\left[\operatorname{tr} \pi_{\lambda}\left(x^{r}\right)\right] x_{r}\right)
$$

and

$$
\begin{aligned}
I_{m}(\lambda)=\left(-\frac{1}{2}\right)^{m} \sum_{i, j, \ldots, k}(\operatorname{tr}[ & \left.\pi_{\lambda}\left(x_{i}\right) \pi_{\lambda}\left(x_{j}\right) \ldots \pi_{\lambda}\left(x_{k}\right)\right] x^{i} x^{j} \ldots x^{k} \\
& +\operatorname{tr}\left[\pi_{\lambda}\left(x_{i}\right) \pi_{\lambda}\left(x_{j}\right) \ldots \pi_{\lambda}\left(x^{k}\right)\right] x^{i} x^{j} \ldots x_{k} \\
& \left.+\ldots+\operatorname{tr}\left[\pi_{\lambda}\left(x^{i}\right) \pi_{\lambda}\left(x^{j}\right) \ldots \pi_{\lambda}\left(x^{k}\right)\right] x_{i} x_{j} \ldots x_{k}\right),
\end{aligned}
$$

where the sum is over all sets of $m$ integers $i, j, \ldots, k$ satisfying $1 \leqslant i, j, \ldots, k \leqslant l$.

Let us now choose a basis of $L$ to be $\left\{h_{1}, \ldots, h_{m} ; x_{\alpha}, x^{\alpha} ; \alpha \in \Phi^{+}\right\}$where $x_{\alpha}$ is a non-zero element of the root space $L_{\alpha}$ and $x^{\alpha}$ is the unique element of the root space $L_{-\alpha}$ which is dual to $x_{\alpha}$ under the Killing form of $L$. Then the dual basis is $\left\{h^{1}, \ldots, h^{m} ; x_{\alpha}, x^{\alpha} ; \alpha \in \Phi^{+}\right\}$. With this choice of basis for $L$ the matrix $A$ may be written:

$$
A=-\frac{1}{2} \sum_{i=1}^{m}\left(\pi_{\lambda}\left(h_{i}\right) h^{i}+\pi_{\lambda}\left(h^{i}\right) h_{i}\right)-\sum_{\alpha \in \Phi^{+}}\left(\pi_{\lambda}\left(x_{\alpha}\right) x^{\alpha}+\pi_{\lambda}\left(x^{\alpha}\right) x_{\alpha}\right) .
$$


Suppose now we choose a basis of $V(\lambda)$ consisting of weight space elements. Since the elements $x_{\alpha}, x^{\beta}$ shift the weights occurring in $V(\lambda)$ the matrices $\pi_{\lambda}\left(x_{\alpha}\right)$ and $\pi_{\lambda}\left(x^{\beta}\right)$ contains no diagonal entries. In fact these matrices are nilpotent endomorphisms of $V(\lambda)$ and a basis for $V(\lambda)$ may be chosen so that the matrices $\pi_{\lambda}\left(x_{\alpha}\right)$ are strictly upper triangular while the $\pi_{\lambda}\left(x^{\beta}\right)$ are strictly lower triangular. On the other hand, the matrices $\pi_{\lambda}\left(h_{i}\right)$ and $\pi_{\lambda}\left(h^{i}\right)$ are diagonal.

In the bases considered above we see that the diagonal entries of the matrix operator $A$ must consist of elements of the Cartan subalgebra $H$ and we may write

$$
A_{j}^{j}=-\frac{1}{2} \sum_{i=1}^{m}\left(\pi_{\lambda}\left(h_{i}\right)_{j}^{j} h^{i}+\pi_{\lambda}\left(h^{i}\right)_{j}^{j} h_{i}\right)
$$

It should be noted that if $T$ is an irreducible contragredient tensor operator of weight $\lambda$ then the above choice of basis for $V(\lambda)$ is equivalent to choosing components $T^{i}$ of $T$ which are weight space elements under the action of $L$ defined by (2). Now suppose $\left\{\lambda_{1}, \ldots, \lambda_{r}\right\} r(=\operatorname{dim} V(\lambda))$ are the weights (not necessarily distinct) occurring in $V(\lambda)$ and suppose the component $T^{i}$ has weight $-\lambda_{i}$ so that

$$
\left[h, T^{i}\right]=-\lambda_{i}(h) T^{i}, \quad h \in H .
$$

Comparing this with equation (2) gives

$$
\pi_{\lambda}(h)_{j}^{i}=\delta_{j}^{i} \lambda_{j}(h) .
$$

Hence we may write

$$
A_{j}^{j}=-\frac{1}{2} \sum_{i=1}^{m}\left(\lambda_{j}\left(h_{i}\right) h^{i}+\lambda_{j}\left(h^{i}\right) h_{i}\right)
$$

Therefore the first-order Casimir may be written in the form

$$
I_{1}(\lambda)=-\frac{1}{2} \sum_{j=1}^{r} \sum_{i=1}^{m}\left(\lambda_{j}\left(h_{i}\right) h^{i}+\lambda_{j}\left(h^{i}\right) h_{i}\right)
$$

where the sum on $j$ is over all weights $\lambda_{j}$ occurring in $V(\lambda)$ (possibly with multiplicities).

For semi-simple Lie algebras this operator is necessarily zero. However this is not so for arbitrary reductive Lie algebras. For a reductive Lie algebra we may express $I_{1}(\lambda)$ in terms of the labelling operator as follows.

On an irreducible representation of a reductive Lie algebra with highest weight $\mu$, $I_{1}(\lambda)$ takes the constant value

$$
I_{1}(\lambda)=-\sum_{j=1}^{k} n(j)\left(\lambda_{j}, \mu\right)
$$


where the sum on $j$ is over the distinct weights occurring in $V(\lambda)$ and $n(j)$ is the multiplicity of the weight $\lambda_{j}$. Hence we may write

$$
I_{1}(\lambda)=-\sum_{j=1}^{k} n(j)\left(\lambda_{j}, \Lambda\right)
$$

This is a generalization of equation (3) appearing in the work of Green [6].

Now let us consider the second-order Casimir $I_{2}(\lambda)$ and the operators $\left(A^{2}\right)_{i}^{i}$. Acting on a maximal weight vector $v_{0}$ of $L$ with highest weight $\mu$ the operator $\left(A^{2}\right)_{i}^{i}$ takes the constant value (see Appendix $A$ )

$$
\left(A^{2}\right)_{i}^{i} v_{0}=\left[\left(\lambda_{i}, \mu\right)^{2}+\sum_{\alpha \in \Phi^{+}}\left[\pi_{\lambda}\left(x^{\alpha}\right) \pi_{\lambda}\left(x_{\alpha}\right)\right]_{i}^{i} \mu\left(t_{\alpha}\right)\right] v_{0},
$$

where

$$
t_{\alpha}=\left[x_{\alpha}, x^{\alpha}\right] \text {. }
$$

Hence we may express the second-order Casimir $I_{2}(\lambda)$ in terms of the labelling operator $\Lambda$ by writing

$$
I_{2}(\lambda)=\sum_{i=1}^{k} n(i)\left(\lambda_{i}, \Lambda\right)^{2}+\sum_{\alpha \in \Phi^{+}} \operatorname{tr}\left[\pi_{\lambda}\left(x^{\alpha}\right) \pi_{\lambda}\left(x_{\alpha}\right)\right] \Lambda\left(t_{\alpha}\right) .
$$

We note here that formulae (33) and (34) are in actual fact independent of our choice of basis for $L$ and $V(\lambda)$.

We may make formulae (33) and (34) fully explicit by using the KostantSteinberg [12] formula for multiplicities and a well-known formula [16] for calculating traces on weight spaces.

Note that, although we have defined the determinant of the matrix $A$ to be the product of its eigenviaues $\Lambda_{i}$, the trace of the matrix $A$ is not in general the sum of its eigenvalues.

The method just considered for evaluating the Casimirs $I_{m}(\lambda)$ unfortunately becomes complicated for higher values of $m$. There is, however, a more systematic way of evaluating the Casimirs $I_{m}(\lambda)$. From equations (27), we see that if $p(x)$ is any polynomial over $\tilde{Z}$, we may write

$$
\begin{aligned}
& \operatorname{tr}_{\lambda}[p(A)]=\sum_{i=1}^{k} p\left(\Lambda_{i}\right) \operatorname{tr}_{\lambda} E_{i}, \\
& \operatorname{tr}_{\lambda}[p(A)]=\sum_{i=1}^{k} p\left(\bar{\Lambda}_{i}\right) \operatorname{tr}_{\lambda} E_{i} .
\end{aligned}
$$

Hence the problem of evaluating the traces of polynomials in $A$ and $A$ and in particular the Casimirs $I_{m}(\lambda)$ reduces to the problem of evaluating the traces of the operators $E_{i}$ and $E_{i}$. 
This problem was first solved for the Lie algebras $O(n), S p(2 n)$ and $U(n)$ by Green [7]. Recently a more systematic approach for evaluating these traces has been developed by Edwards [4] and Okubo [15].

Suppose $V(\mu)$ is an irreducible representation of $L$ with highest weight $\mu$. Then acting on $V(\mu)$ the operator $A$ may be written

$$
A=-\frac{1}{2} \sum_{r=1}^{l}\left(\pi_{\lambda}\left(x_{r}\right) \pi_{\mu}\left(x^{r}\right)+\pi_{\lambda}\left(x^{r}\right) \pi_{\mu}\left(x_{r}\right)\right) .
$$

As remarked earlier, $A$ may be regarded as an operator on the tensor product representation $V(\lambda) \otimes V(\mu)$. Let us now consider the total trace of $A$ with respect to both the $\lambda$ and $\mu$ indices which occur. We denote this trace by $\operatorname{tr}_{\lambda \otimes \mu} A$. It can be shown (details in Appendix B) that the total trace of powers of $A$ are related to the Casimirs $I_{m}(\lambda)$ by the formula

$$
\operatorname{tr}_{\lambda \otimes \mu}\left(A^{m}\right)=I_{m}(\lambda) \operatorname{Dim} V(\mu) .
$$

More generally, if $p(x) \in \tilde{Z}[x]$ then

$$
\operatorname{tr}_{\lambda \otimes \mu} p(A)=\operatorname{tr}_{\lambda} p(A) \operatorname{Dim} V(\mu)
$$

and, in particular,

$$
\operatorname{tr}_{\lambda \otimes \mu} E_{i}=\operatorname{tr}_{\lambda} E_{i} \operatorname{Dim} V(\mu) .
$$

Following the notation of Section 2, let us write the Clebsch-Gordan decomposition of $V(\lambda) \otimes V(\mu)$ in the form

$$
V(\lambda) \otimes V(\mu)=\bigoplus_{i=1}^{k} n(i) V\left(\mu+\lambda_{i}\right),
$$

where $n(i)$ denotes the multiplicity of the weight $\lambda_{i}$ in $V(\lambda)$. It was shown in Section 2 that on each space $V\left(\mu+\lambda_{i}\right)$ the operator $A$ takes the constant value $\Lambda_{i}$ which is given by equation (17) with the labelling operator $\Lambda$ replaced by the weight $\mu$.

More generally we may show by induction that if $p(x)$ is any polynomial over $\tilde{Z}$ then on the space $V\left(\mu+\lambda_{i}\right)$ the operator $p(A)$ takes the constant value $p\left(\Lambda_{i}\right)$. It follows immediately that

$$
\operatorname{tr}_{\lambda \otimes \mu} p(A)=\sum_{i=1}^{k} n(i) p\left(\Lambda_{i}\right) \operatorname{Dim} V\left(\mu+\lambda_{i}\right)
$$

In particular we obtain

$$
\operatorname{tr}_{\lambda \otimes \mu} E_{i}=n(i) \operatorname{Dim} V\left(\mu+\lambda_{i}\right) .
$$

Comparing equations (36) and (37) then gives

$$
\mathbf{t r}_{\lambda} E_{\imath}=n(l) \frac{\operatorname{Dim} V\left(\mu+\lambda_{i}\right)}{\operatorname{Dim} V(\mu)} .
$$


Substituting in Weyl's dimension formula gives the result

$$
\operatorname{tr}_{\lambda} E_{i}=n(i) \prod_{\alpha \in \Phi^{+}} \frac{\left(\mu+\delta+\lambda_{i}, \alpha\right)}{(\mu+\delta, \alpha)}
$$

In a similar way we may show that

$$
\operatorname{tr}_{\lambda} E_{i}=n(i) \prod_{\alpha \in \Phi^{+}} \frac{\left(\mu+\delta-\lambda_{i}, \alpha\right)}{(\mu+\delta, \alpha)} .
$$

Using our labelling operator we may now write

$$
\begin{aligned}
& \operatorname{tr}_{\lambda} E_{i}=n(i) \prod_{\alpha \in \Phi^{+}} \frac{\left(\Lambda+\delta+\lambda_{i}, \alpha\right)}{(\Lambda+\delta, \alpha)} \\
& \operatorname{tr}_{\lambda} E_{i}=n(i) \prod_{\alpha \in \Phi^{+}} \frac{\left(\Lambda+\delta-\lambda_{i}, \alpha\right)}{(\Lambda+\delta, \alpha)} .
\end{aligned}
$$

From equations (35) and (38) we may now evaluate the traces of arbitrary polynomials in $A$ and $A$.

\section{Reduction of the identity}

We have already shown in Section 2 that the matrix operator $A$ satisfies a certain polynomial identity. However, in a particular irreducible representation $A$ may satisfy a reduced polynomial equation of lower degree. It is our aim in this section to obtain the minimum polynomial identity satisfied by the matrix $A$ on a given finite dimensional irreducible representation $V(\mu)$.

From the remarks in the previous section we see that the matrix $A$, in a given finite dimensional irreducible representation $V(\mu)$ of highest weight $\mu$, may be regarded as an operator on the tensor product representation $V(\lambda) \otimes V(\mu)$. The reduced identity satisfied by the matrix $A$ is in fact related to the Clebsch-Gordan decomposition of $V(\lambda) \otimes V(\mu)$. In general we may write the Clebsch-Gordan decomposition of $V(\lambda) \otimes V(\mu)$ in the form

$$
V(\lambda) \otimes V(\mu)=\bigoplus_{i=1}^{k} n(i) V\left(\mu+\lambda_{i}\right),
$$

where $\left\{\lambda_{1}, \ldots, \lambda_{k}\right\}$ are the distinct weights occurring in $V(\lambda)$ and $n(i)$ is the multiplicity of the weight $\lambda_{i}$. However, for a given irreducible representation $V(\mu)$ not all of the representations $V\left(\mu+\lambda_{i}\right)$ will appear in the Clebsch-Gordan series.

We shall now determine precisely which irreducible representations occur in the Clebsch-Gordan decomposition of $V(\lambda) \otimes V(\mu)$ and hence determine the reduced identity of the operator $A$. 
It is known (see, for example, Humphreys [10]) that if $V(\mu)$ is a finite dimensional irreducible representation of a semi-simple Lie algebra $L$ with highest weight $\mu$ and $\alpha \in \Phi+$ then

$$
(\mu, \alpha) \geqslant 0
$$

We shall use this fact to prove the following result.

Proposition. Let $\left\{\lambda_{1}, \ldots, \lambda_{k}\right\}$ be the distinct weights occurring in $V(\lambda)$. Then the irreducible representation $V\left(\mu+\lambda_{i}\right)$ will occur in the Clebsch-Gordan decomposition of $V(\lambda) \otimes V(\mu)$ if and only if $\left(\mu+\delta+\lambda_{i}, \alpha\right) \neq 0$ for every $\alpha$ in $\Phi^{+}$.

Proof. Suppose $V\left(\mu+\lambda_{i}\right)$ occurs in the Clebsch-Gordan series. Since $V\left(\mu+\lambda_{i}\right)$ is finite dimensional and irreducible we see that $\left(\mu+\lambda_{i}, \alpha\right) \geqslant 0$ for every $\alpha$ in $\Phi^{+}$. Hence if $\alpha \in \Phi^{+}$then $\left(\mu+\lambda_{i}+\delta, \alpha\right)$ must be non-zero otherwise we would obtain

$$
\left(\mu+\lambda_{i}, \alpha\right)=-(\delta, \alpha)<0
$$

Conversely suppose $\left(\mu+\delta+\lambda_{i}, \alpha\right)$ is non-zero for every $\alpha$ in $\Phi^{+}$. We prove that $V\left(\mu+\lambda_{i}\right)$ must occur in the Clebsch-Gordan decomposition of $V(\lambda) \otimes V(\mu)$ by a contradiction argument. Suppose $V\left(\mu+\lambda_{i}\right)$ does not occur in the Clebsch-Gordan series. The associated projection operator $E_{i}$ must vanish on $V(\mu)$. In particular, the $\lambda$-trace of $E_{i}$ must vanish and, from formula (38), this implies that $\left(\mu+\delta+\lambda_{i}, \alpha\right)=0$ for some $\alpha$ in $\Phi^{+}$, a contradiction.

Thus $V\left(\mu+\lambda_{i}\right)$ must occur in the Clebsch-Gordan decomposition of $V(\lambda) \otimes V(\mu)$ and the result is proved.

Now let $I(\lambda, \mu)$ denote the index set

$$
\left\{i ;\left(\lambda_{i}+\mu+\delta, \alpha\right) \neq 0 \text { for all } \alpha \in \Phi+\right\} .
$$

Then we may write the Clebsch-Gordan decomposition of $V(\lambda) \otimes V(\mu)$ in the form

$$
V(\lambda) \otimes V(\mu)=\underset{i \in I(\lambda, \mu)}{\oplus} n(i) V\left(\mu+\lambda_{i}\right)
$$

Suppose now that $\tilde{T}$ is an irreducible tensor operator of highest weight $\lambda$. Since $\tilde{T}_{[i]}=\tilde{T} E_{i}$ we see that the shift tensor $\tilde{T}_{[i]}$ vanishes on $V(\mu)$ if and only if $E_{i}$ vanishes on $V(\mu)$. Hence on the space $V(\mu)$, we may write the decompositon of the tensor operator $\tilde{T}$ into its distinct shift components in the form

$$
\tilde{T}=\sum_{i \in I(\lambda, \mu)} \tilde{T}_{[i]}
$$

This states that the number of linearly independent tensor operators, transforming with an irreducible representation $V(\lambda)$, when acting on an irreducible 
representation $V(\mu)$ is

$$
\sum_{i \in I(\lambda, \mu)} n(i)
$$

This sharpens the result obtained by Baird and Biedenharn [1].

In a similar way we may show that the operator $E_{i}$ vanishes on $V(\mu)$ if and only if $\left(\mu+\delta-\lambda_{i}, \alpha\right)=0$ for some $\alpha \in \Phi^{+}$. Hence we may resolve a contragredient tensor $T$ into its shift components $T_{[i]}$ by writing

$$
\boldsymbol{T}=\sum_{i \in \tilde{I}(\lambda, \mu)} T_{[i]}
$$

where

$$
\tilde{I}(\lambda, \mu)=\left\{i ;\left(\mu+\delta-\lambda_{i}, \alpha\right) \neq 0 \text { for all } \alpha \in \Phi^{+}\right\} .
$$

Then on the space $V(\mu)$ the number of linearly independent contragredient tensor operators transforming with an irreducible representation $V(\lambda)$ is

$$
\sum_{i \in \overline{\bar{I}}(\lambda, \mu)} n(i) \text {. }
$$

From these remarks we see therefore that in an irreducible representation $V(\mu)$ the matrices $A$ and $A$ satisfy the reduced identities

$$
\prod_{i \in I(\lambda, \mu)}\left(A-\frac{1}{2}(\lambda, \lambda+2 \delta)+\frac{1}{2}\left(\lambda_{i}, 2(\mu+\delta)+\lambda_{i}\right)\right)=0
$$

and

$$
\prod_{i \in \bar{T}(\lambda, \mu)}\left(\bar{A}-\frac{1}{2}(\lambda, \lambda+2 \delta)-\frac{1}{2}\left(\lambda_{i}, 2(\mu+\delta)-\lambda_{i}\right)\right)=0 .
$$

Clearly these identities are the minimal polynomial identities satisfied by $A$ and $A$ on $V(\mu)$ and may be regarded as a generalization of the reduced identities discovered by Green [6] for the special case of $G L(n)$.

\section{Commutation relations}

In this section we shall be primarily concerned with a semi-simple Lie algebra $L$ which is embedded in a larger Lie algebra $K$. From Weyl's theorem $K$ must be a totally reducible representation of $L$ under the adjoint action of $L$ and hence we have a decomposition

$$
L \oplus T=K,
$$

where $T$ is a tensor operator of $L ;[L, T] \subseteq T$.

If $L_{0}$ denotes the subspace of $K$ consisting of invariant operators of $L$ we may assume that

$$
[T, T] \subseteq L \oplus L_{0}
$$


This is the usual situation which occurs in the state labelling problems and covers most situations of interest in physics.

In view of equation (39) we shall henceforth refer to a tensor operator (respectively contragredient tensor operator) as an operator with components $T_{i}$ (respectively $T^{i}$ ) which transform according to (1) (respectively (2)) and also satisfy the commutation relations

$$
\left[T_{i}, T_{j}\right]=x_{i j}+c_{i j}
$$

where $x_{i j}$ belongs to $L$ and $c_{i j}$ is an invariant operator of the Lie algebra.

Given these commutation relations for tensor operators it is of interest to determine the commutation relations satisfied by their shift components. The characteristic identities have recently been applied to the labelling problems $O(n) \subset U(n)$ and $U(m) \subset S p(2 m)$ by Green, Hurst and Ilamed [8] and their approach relied heavily on a knowledge of such commutation relations. It is our aim to show how the characteristic identities may be applied to evaluate such commutation relations in more general situations.

Following our previous notation let $T$ be an irreducible contragredient tensor operator of weight $\lambda$ and let $A$ be the matrix as defined by (3). Finally let $\left\{\lambda_{1}, \ldots, \lambda_{k}\right\}$ be the distinct weights occurring in $V(\lambda)$ and suppose $T_{[i]}$ is the shift component of $T$ corresponding to the weight $\lambda_{i}$.

From equations (12) and (17) we may write the roots $\Lambda_{i}$ of the identity (16) in the form

$$
\begin{aligned}
\Lambda_{i}=\frac{1}{2} \pi_{\lambda}\left(c_{L}\right) & -\frac{1}{2} \sum_{j=1}^{m}\left(\lambda_{i}\left(h_{j}\right) \Lambda\left(h^{j}\right)+\Lambda\left(h_{j}\right) \lambda_{i}\left(h^{\jmath}\right)\right) \\
& -\frac{1}{2}\left(\lambda_{i}, \lambda_{i}\right)-\frac{1}{2} \sum_{\alpha \in \Phi^{+}} \lambda_{i}\left(t_{\alpha}\right) .
\end{aligned}
$$

From equation (10) we immediately obtain

$$
\left[\Lambda_{i}, T_{[j]}\right]=\left(\lambda_{i}, \lambda_{j}\right) T_{[j]}
$$

Now let us introduce the weight matrix $\left(M_{i j}\right)=\left(\left(\lambda_{i}, \lambda_{j}\right)\right)$. We may therefore write

and hence

$$
\left[\Lambda_{i}, T_{[j]}\right]=M_{i j} T_{[j]}
$$

$$
\left[\Lambda_{i}, T\right]=\sum_{j=1}^{k} M_{i j} T_{[j]}
$$

If the matrix $M$ is invertible we may invert equation (42) by writing

$$
T_{[j]}=\sum_{i} M_{j i}^{-1}\left[\Lambda_{i}, T\right]
$$


In such a situation we have, using (43),

$$
\left[T_{[j]}^{\alpha}, T^{\beta}\right]=M_{j i}^{-1}\left[\left[\Lambda_{i}, T^{\alpha}\right], T^{\beta}\right]
$$

which, from the Jacobi identity, may in turn be written

$$
\left[T_{[j]}^{\alpha}, T^{\beta}\right]=-M_{j i}^{-1}\left[\left[T^{\beta}, \Lambda_{i}\right], T^{\alpha}\right]-M_{j i}^{-1}\left[\left[T^{\alpha}, T^{\beta}\right], \Lambda_{i}\right] .
$$

If $c$ is an invariant operator for the Lie algebra $L$ we see that $c$ cannot alter the weight of an irreducible representation of $L$ so that we may regard $c$ as commuting with the labelling operator $\Lambda$. Hence we have $\left[c, \Lambda_{i}\right]=0$ and, in view of the commutation relations (40), we may write

$$
\left[\left[T^{\alpha}, T^{\beta}\right], \Lambda_{i}\right]=0 .
$$

Hence we have

$$
\begin{aligned}
{\left[T_{[j]}^{\alpha}, T^{\beta}\right] } & =-M_{j i}^{-1}\left[\left[T^{\beta}, \Lambda_{i}\right], T^{\alpha}\right] \\
& =\left[T_{[j]}^{\beta}, T^{\alpha}\right] .
\end{aligned}
$$

Therefore $\left[T_{[j]}^{\alpha}, T^{\beta}\right]$ is symmetric in $\alpha$ and $\beta$ and it follows that

$$
\left[T^{\alpha}, T^{\beta}\right]=\sum_{j=1}^{k}\left[T_{[j]}^{\alpha}, T^{\beta}\right]
$$

is also symmetric in $\alpha$ and $\beta$. On the other hand, $\left[T^{\alpha}, T^{\beta}\right]=-\left[T^{\beta}, T^{\alpha}\right]$ is obviously antisymmetric in $\alpha$ and $\beta$ from which it must follow

$$
\left[T^{\alpha}, T^{\beta}\right]=0 .
$$

A similar argument holds for tensor operators $\widetilde{T}$.

Hence we have proved that if $V(\lambda)$ is an irreducible representation of $L$ with distinct weights $\left\{\lambda_{1}, \ldots, \lambda_{k}\right\}$ and whose tensor matrix $M_{i j}=\left(\lambda_{i}, \lambda_{j}\right)$ is invertible then any tensor operator (in the sense of equation (40)) of weight $\lambda$ necessarily has commuting components.

For example, this result states that any vector operator for $U(n)$ must in such circumstances have commuting components.

Wi remark here that the converse of this result does not hold in general (consider the labelling problem $U(2) \subset S p(4)$ for example). The tensor matrix may be regarded as a generalization of the Cartan matrix for semi-simple Lie algebras. However, unlike the Cartan matrix the tensor matrix will be singular in many cases. For example, if the distinct weights occurring in $V(\lambda)$ are linearly dependent elements of $H^{*}$ then the determinant of the tensor matrix $M$ must vanish since we will have linearly dependent rows. Such a situation must occur when the number of distinct weights exceeds the dimension of the Cartan subalgebra. 
Suppose $T$ is a contragredient tensor operator with components $T^{\alpha}$. From the commutation relations (40) we may write

$$
\left[T^{\alpha}, T^{\beta}\right]=x+c
$$

where $x$ is an element of the Lie algebra $L$ and $c$ is an invariant of $L$. We conclude by showing how the characteristic identities may be applied to the evaluation of commutators $T_{[i]}^{\alpha}, T_{[j]}^{\beta}$ in certain special cases.

Resolving $T$ into its distinct shift components $T_{[i]}$ allows us to write

$$
\sum_{i, j=1}^{k}\left[T_{[i]}^{\alpha}, T_{[j]}^{\beta}\right]=x+c .
$$

Note that each commutator $\left[T_{[k]}^{\alpha}, T_{[l]}^{\beta}\right]$ has shift weight $\lambda_{k}+\lambda_{l}$ since, for every $h$ in $H$, we have

$$
\left[\Lambda(h),\left[T_{[k]}^{\alpha}, T_{[l]}^{\beta}\right]\right]=-\left(\lambda_{k}+\lambda_{l}\right)(h)\left[T_{[k]}^{\alpha}, T_{[l]}^{\beta}\right] .
$$

Separating out terms shifting from one irreducible representation to another and noting that the right-hand side of (44) has shift zero we have, for each $\lambda_{i}+\lambda_{j} \neq 0$,

$$
\sum_{\substack{p, q \\ \lambda_{p}+\lambda_{p}=\lambda_{i}+\lambda_{j}}}\left[T_{[p]}^{\alpha}, T_{[q]}^{\beta}\right]=0 .
$$

Note that the commutators $\left[T_{[i]\}}^{\alpha}, T_{[j]}^{\beta}\right]$ and $\left[T_{[j]}^{\alpha}, T_{[i]}^{\beta}\right]$ both necessarily occur in this sum. When these are the only two commutators occurring in this sum (which always occurs when the tensor matrix is invertible) we may write

or

$$
\left[T_{[i]}^{\alpha}, T_{[j]}^{\beta}\right]+\left[T_{[j]\}}^{\alpha}, T_{[i]}^{\beta}\right]=0
$$

$$
\left[T_{[i]}^{\alpha}, T_{[j]}^{\beta}\right]=\left[T_{[i]}^{\beta}, T_{[j]}^{\alpha}\right] .
$$

In this case the commutators $\left[T_{[i]}^{\alpha}, T_{[j]}^{\beta}\right]$ may be evaluated. When $i=j$ we must have

$$
\left[T_{[i]}^{\alpha}, T_{[i]}^{\beta}\right]=0 .
$$

In the case when $i \neq j$ we may evaluate the commutators by a simple application of the characteristic identity. Now

$$
\left(A-\Lambda_{i}\right)_{\gamma}^{\alpha}\left[T_{[i]}^{\gamma}, T_{[j]}^{\beta}\right]=\left[\left(A-\Lambda_{i}\right)_{\gamma}^{\alpha} T_{[i]}^{\gamma}, T_{[j]}^{\beta}\right]-\left[\left(A-\Lambda_{i}\right)_{\gamma}^{\alpha}, T_{[j]}^{\beta}\right] T_{[i]}^{\gamma}
$$

From equation (13) the first commutator on the right must vanish and we obtain

$$
\left(A-\Lambda_{i}\right)_{\gamma}^{\alpha}\left[T_{[i]}^{\gamma}, T_{[j]}^{\beta}\right]=\left(\lambda_{i}, \lambda_{j}\right) T_{[j]}^{\beta} T_{[i]}^{\alpha}-\left[A_{\gamma}^{\alpha}, T_{[j]}^{\beta}\right] T_{[i]}^{\gamma} \text {. }
$$

On the other hand, the symmetry condition (45) gives

$$
\begin{aligned}
\left(A-\Lambda_{j}\right)_{\gamma}^{\alpha}\left[T_{[i]}^{\gamma}, T_{[j]}^{\beta}\right) & =\left(A-\Lambda_{j}\right)_{\gamma}^{\alpha}\left[T_{[i]}^{\beta}, T \gamma_{j j}\right] \\
& \left.=-\left(\lambda_{i}, \lambda_{j}\right) T_{[i]}^{\beta} T_{[j]}^{\alpha}+\left[A_{\gamma}^{\alpha}, T_{[i]}^{\beta}\right] T\right\}_{j]} .
\end{aligned}
$$


Subtracting (47) from (46) then gives

$$
\begin{aligned}
\left(\Lambda_{j}-\Lambda_{i}\right)\left[T_{[i]}^{\alpha}, T_{[j]}^{\beta}\right]=\left(\lambda_{i}, \lambda_{j}\right) & \left(T_{[i]}^{\alpha} T_{[j]}^{\beta}+T_{[i]}^{\alpha} T_{[j]}^{\beta}\right) \\
& -\left(\left[A_{\gamma}^{\alpha}, T_{[j]}^{\beta}\right] T_{[i]}^{\gamma_{i]}}+\left[A_{\gamma}^{\alpha}, T_{[i]}^{\beta}\right] T_{[j]}^{\gamma}\right) .
\end{aligned}
$$

Using (2) and the definition of the matrix $A$ we may simplify the second term appearing on the right of equation (48) by writing

$$
\begin{aligned}
{\left[A_{\gamma}^{\alpha}, T_{[i]}^{\beta}\right] T[j] } & \left.+\left[A_{\gamma}^{\alpha}, T f_{[i]}^{\beta}\right] T\right\}_{j]}=\frac{1}{2} \sum_{r}\left(\left[x^{r}, T_{[j]}^{\beta}\right]\left[x_{r}, T_{[i]}^{\alpha}\right]+\left[x_{r}, T_{[j]}^{\beta}\right]\left[x^{r}, T_{[i]}^{\alpha}\right]\right. \\
& \left.+\left[x^{r}, T_{[i]}^{\beta}\right]\left[x_{r}, T_{[j]}^{\alpha}\right]+\left[x_{r}, T_{[i]}^{\beta}\right]\left[x^{r}, T_{[j]}^{\alpha}\right]\right) .
\end{aligned}
$$

A simple calculation shows that

$$
\left.\sum_{r}\left(\left[x_{r}, T_{[i]}^{\beta}\right]\left[x^{r}, T_{[i]}^{\alpha}\right]+\left[x^{r}, T_{[j]}^{\beta}\right] x_{r}, T_{[i]}^{\alpha}\right]\right)=\sum_{r}\left[x_{r},\left[x^{r}, T_{[j]}^{\beta} T_{[i]}^{\alpha}\right]\right]-2 \pi_{\lambda}\left(c_{L}\right) T_{[j]}^{\beta} T_{[i]}^{\alpha} .
$$

Hence the right-hand side of equation (49) may be written

$$
\left(\frac{1}{2} \bar{c}_{L}-\pi_{\lambda}\left(c_{L}\right)\right)\left(T_{[i]}^{\alpha} T_{[j]}^{\beta}+T_{[j]}^{\alpha} T_{[i]}^{\beta}\right)
$$

where $\bar{c}_{L}$ is the universal Casimir element for the tensor $T_{[i]} T_{[j]}+T_{[j]} T_{[i,}$,

$$
\bar{c}_{L} \cdot\left(T_{[i]} T_{[j]}+T_{[j]} T_{[i]}\right)=\sum_{r}\left[x_{r},\left[x^{r},\left(T_{[i]} T_{[j]}+T_{[j]} T_{[i]}\right)\right]\right] .
$$

Note that when $T_{[i]} T_{[j]}+T_{[j]} T_{[i]}$ is an irreducible contragredient tensor operator, of weight $\nu$ say, then $\bar{c}_{L}$ will take the constant value $\pi_{\nu}\left(c_{L}\right)$. More generally, it is conceivable that $T_{[i]} T_{[j]}+T_{[j]} T_{[i]}$ will not be irreducible in which case it will be decomposable into a sum of irreducible contragredient tensors on which the operator $\bar{c}_{L}$ will take constant values.

Substituting (50) into equation (48) allows us to write

$$
\left(\Lambda_{j}-\Lambda_{i}\right)\left[T_{[i]}^{\alpha}, T_{[j]}^{\beta}\right]=\left(\left(\lambda_{i}, \lambda_{j}\right)+\pi_{\lambda}\left(c_{L}\right)-\frac{1}{2} \bar{c}_{L}\right)\left(T_{[i]} T_{[j]}+T_{[j]} T_{[i]}\right)^{\alpha \beta} \text {. }
$$

The commutator $\left[T_{[i]}^{\alpha}, T_{[j]}^{\beta}\right]$ vanishes when $i=j$ and when $i \neq j$ we have, substituting for $\Lambda_{i}$ and $\Lambda_{j}$ using equation (17),

$$
\left[T_{[i]}^{\alpha}, T \beta_{j]}^{\beta}\right]=2\left(\frac{\left(\lambda_{i}, \lambda_{j}\right)+\pi_{\lambda}\left(c_{L}\right)-\frac{1}{2} \bar{c}_{L}}{\left(\lambda_{i}, \lambda_{i}\right)-\left(\lambda_{j}, \lambda_{j}\right)+\left(\lambda_{i}-\lambda_{j}, 2(\Lambda+\delta)\right)}\right)\left(T_{[i]} T_{[j]}+T_{[j]} T_{[i]}\right)^{\alpha \beta} .
$$

In a similar way, using the adjoint identity, we may apply this procedure to an irreducible tensor operator $\tilde{T}$ to give the commutation relations

and

$$
\left[\tilde{T}_{[i]_{\alpha}}, \tilde{T}_{[i]_{\beta}}\right]=0
$$

$$
\left[\tilde{T}_{[i]_{\alpha}}, \tilde{T}_{\left.[]_{\beta}\right]}\right]=2\left(\frac{\left(\lambda_{i}, \lambda_{j}\right)+\pi_{\lambda}\left(c_{L}\right)-\frac{1}{2} \bar{c}_{L}}{\left(\lambda_{i}, \lambda_{i}\right)-\left(\lambda_{j}, \lambda_{j}\right)+\left(\lambda_{j}-\lambda_{i}, 2(\Lambda+\delta)\right)}\right)\left(\tilde{T}_{[i]} \tilde{T}_{[j]}+\tilde{T}_{[j]} \tilde{T}_{[i]}\right)_{\alpha \beta}
$$


Since this paper was motivated from Green's work [6] on $G L(n)$ we conclude by considering the example $G L(n)$.

The generators $a_{j}^{i}$ of $G L(n)$ satisfy the commutation relations

$$
\left[a_{j}^{i}, a_{l}^{k}\right]=\delta_{l}^{i} a_{j}^{k}-\delta_{j}^{k} a_{l}^{i} .
$$

We take as a C.S.A. the vector space spanned by the operators $a_{i}^{i}$. In this case an element $\lambda$ of $H^{*}$ may be identified with an $n$-tuple $\left(\lambda_{1}, \lambda_{2}, \ldots, \lambda_{n}\right)$ where $\lambda_{r}=\lambda\left(a_{r}^{r}\right)$. We may write our labelling operator $\Lambda$ in the form $\left(\Lambda_{1}, \Lambda_{2}, \ldots, \Lambda_{n}\right)$.

Now let $V(\lambda)$ denote the fundamental vector representation of $G L(n)$ and write $E_{j}^{i}$ for a typical elementary matrix (all entries vanishing except for 1 in the $(i, j)$ position). In this case our matrix operator $A$ may be written

$$
A=\sum_{i, j=1}^{n} E_{j}^{i} \otimes a_{i}^{j}
$$

which is Green's adjoint matrix. On an irreducible representation of $G L(n)$ with highest weight $\left(\lambda_{1}, \ldots, \lambda_{n}\right)$ the matrix $A$ satisfies the polynomial identity

$$
\prod_{r=1}^{n}\left(A+\lambda_{z}-r+1\right)=0 .
$$

A $G L(n)$ vector operator may be defined as an operator with $n$ components $T_{i}$ satisfying

$$
\left[a_{j}^{i}, T_{k}\right]=\delta_{k}^{i} T_{j} .
$$

Such an operator necessarily has commuting components since the tensor matrix of $T$ is $M_{i j}=\delta_{i j}$ which is certainly invertible. From this it follows that $T$ may be resolved into a sum of shift vectors

$$
T=\sum_{r=1}^{n} T_{[r]}
$$

where $T_{[r]}$ increases the eigenvalue of the labelling operator $\Lambda_{r}$ by one unit leaving the other $\Lambda_{k}$ unchanged;

$$
\Lambda_{k} T_{[r]}=T_{[r]}\left(\Lambda_{k}+\delta_{r k}\right) .
$$

From equation (52) it follows that the shift components $T_{[r]}$ of $T$ satisfy the commutation relations

$$
\begin{gathered}
{\left[T_{[r \mathrm{k}}, T_{[r \mathrm{~b}}\right]=0} \\
{\left[T_{[r \mathrm{k}}, T_{[k \mathrm{k}\}}\right]=\left(\Lambda_{r}-\Lambda_{k}+k-r\right)^{-1}\left(T_{[r]} T_{[k]}+T_{[k]} T_{[r]}\right)_{i j}, \quad k \neq r .}
\end{gathered}
$$

In this case the tensor $T_{[r]} T_{[k]}+T_{[k]} T_{[r]}$ is a symmetric two-rank tensor operator and is hence irreducible. It may be further shown that these tensors form the shift components of the symmetric two-rank tensor $T^{i j}=T^{i} \otimes T^{j}$.

A similar analysis may also be carried out for $O(n)$. 


\section{Acknowledgements}

The author would like to thank Professor H. S. Green, Dr. A. L. Carey and S. A. Edwards for several useful discussions. He would also like to acknowledge the financial support of a Commonwealth Postgraduate Research Award.

\section{Appendix A}

Here we evaluate the diagonal entries of the operator $A^{2}$ in the basis considered in Section 3.

We have

$$
\begin{aligned}
\left(A^{2}\right)_{i}^{i}=\sum_{\alpha, \beta \in \Phi^{+}} & \left(\left[\pi_{\lambda}\left(x^{\alpha}\right) \pi_{\lambda}\left(x^{\beta}\right)\right]_{i}^{i} x_{\alpha} x_{\beta}+\left[\pi_{\lambda}\left(x^{\alpha}\right) \pi_{\lambda}\left(x_{\beta}\right)\right]_{i}^{i} x_{\alpha} x^{\beta}\right. \\
& \left.+\left[\pi_{\lambda}\left(x_{\alpha}\right) \pi_{\lambda}\left(x^{\beta}\right)\right]_{i}^{i} x^{\alpha} x_{\beta}+\left[\pi_{\lambda}\left(x_{\alpha}\right) \pi_{\lambda}\left(x_{\beta}\right)\right]_{i}^{i} x^{\alpha} x^{\beta}\right) \\
& +\frac{1}{4} \sum_{k, l=1}^{m}\left(\left[\pi_{\lambda}\left(h^{k}\right) \pi_{\lambda}\left(h^{l}\right)\right]_{i}^{i} h_{k} h_{l}+\left[\pi_{\lambda}\left(h^{k}\right) \pi_{\lambda}\left(h_{l}\right)\right]_{i}^{i} h_{k} h^{l}\right. \\
& \left.+\left[\pi_{\lambda}\left(h_{k}\right) \pi_{\lambda}\left(h^{l}\right)\right]_{i}^{i} h^{k} h_{l}+\left[\pi_{\lambda}\left(h_{k}\right) \pi_{\lambda}\left(h_{l}\right)\right]_{i}^{i} h^{k} h^{l}\right) .
\end{aligned}
$$

We may simplify this expression by noting that if $\alpha, \beta \in \Phi^{+}$then the matrices $\pi_{\lambda}\left(x^{\alpha}\right) \pi_{\lambda}\left(x^{\beta}\right)$ and $\pi_{\lambda}\left(x_{\alpha}\right) \pi_{\lambda}\left(x_{\beta}\right)$ have no diagonal entries. Similarly $\pi_{\lambda}\left(x^{\alpha}\right) \pi_{\lambda}\left(x_{\beta}\right)$ has no diagonal entries unless $\alpha=\beta$. From these considerations and equation (30) we may write

$$
\begin{aligned}
\left(A^{2}\right)_{i}^{i}=\sum_{\alpha \in \Phi^{+}}\left(\left[\pi_{\lambda}\left(x_{\alpha}\right) \pi_{\lambda}\left(x^{\alpha}\right)\right]_{i}^{i} x^{\alpha} x_{\alpha}+\left[\pi_{\lambda}\left(x^{\alpha}\right) \pi_{\lambda}\left(x_{\alpha}\right)\right]_{i}^{i} x_{\alpha} x^{\alpha}\right) \\
+\frac{1}{4} \sum_{k l=1}^{m}\left(\lambda_{i}\left(h^{k}\right) \lambda_{i}\left(h^{\top}\right) h_{k} h_{l}+\lambda_{i}\left(h^{k}\right) \lambda_{i}\left(h_{l}\right) h_{k} h^{l}\right. \\
\left.+\lambda_{i}\left(h_{k}\right) \lambda_{i}\left(h^{\top}\right) h^{k} h_{l}+\lambda_{i}\left(h_{k}\right) \lambda_{i}\left(h_{l}\right) h^{k} h^{\top}\right) .
\end{aligned}
$$

Hence acting on a maximal weight vector $v_{0}$ of weight $\mu$ we obtain

$$
\left(A^{2}\right)_{i}^{i} v_{0}=\left(\lambda_{i}, \mu\right)^{2} v_{0}+\sum_{\alpha \in \Phi^{+}}\left[\pi_{\lambda}\left(x^{\alpha}\right) \pi_{\lambda}\left(x_{\alpha}\right)\right]_{i}^{i} \mu\left(t_{\alpha}\right) v_{0}
$$

where

$$
t_{\alpha}=\left[x_{\alpha}, x^{\alpha}\right]
$$

\section{Appendix B}

Here we calculate the eigenvalues of the Casimir invariants $I_{m}(\lambda)$ in terms of the total trace of the operator $A^{m}$ when acting on an irreducible representation $V(\mu)$ of $L$ with highest weight $\mu$. 
Acting on $V(\mu)$ we may write

$$
\begin{aligned}
A^{m}=\left(-\frac{1}{2}\right)^{m} \sum_{\mathbf{k}}[ & \pi_{\lambda}\left(x^{k_{1}}\right) \pi_{\lambda}\left(x^{k_{2}}\right) \ldots \pi_{\lambda}\left(x^{k_{m}}\right) \pi_{\mu}\left(x_{k_{1}}\right) \pi_{\mu}\left(x_{k_{2}}\right) \ldots \pi_{\mu}\left(x_{k_{m}}\right) \\
& +\pi_{\lambda}\left(x^{k_{1}}\right) \pi_{\lambda}\left(x^{k_{2}}\right) \ldots \pi_{\lambda}\left(x_{k_{m}}\right) \pi_{\mu}\left(x_{k_{1}}\right) \pi_{\mu}\left(x_{k_{2}}\right) \ldots \pi_{\mu}\left(x^{k_{m}}\right) \\
& +\ldots \ldots \\
& \left.+\pi_{\lambda}\left(x_{k_{1}}\right) \pi_{\lambda}\left(x_{k_{2}}\right) \ldots \pi_{\lambda}\left(x_{k_{m}}\right) \pi_{\mu}\left(x^{k_{1}}\right) \ldots \pi_{\mu}\left(x^{k_{m}}\right)\right]
\end{aligned}
$$

where the sum is over integers $k_{i}$ from 1 to $l$. Hence we obtain

$$
\begin{aligned}
\operatorname{tr}_{\lambda \otimes \mu}\left(A^{m}\right)=\left(-\frac{1}{2}\right)^{m} & \sum_{\mathbf{k}}\left[\operatorname{tr}_{\lambda}\left(\pi_{\lambda}\left(x^{k_{1}}\right) \pi_{\lambda}\left(x^{k_{2}}\right) \ldots \pi_{\lambda}\left(x^{k_{m}}\right)\right) \operatorname{tr}_{\mu}\left(\pi_{\mu}\left(x_{k_{1}}\right) \ldots \pi_{\mu}\left(x_{k_{m}}\right)\right)\right. \\
& +\operatorname{tr}_{\lambda}\left(\pi_{\lambda}\left(x^{k_{1}}\right) \pi_{\lambda}\left(x^{k_{2}}\right) \ldots \pi_{\lambda}\left(x_{k_{m}}\right)\right) \operatorname{tr}_{\mu}\left(\pi_{\mu}\left(x_{k_{1}}\right) \ldots \pi_{\mu}\left(x^{k_{m}}\right)\right) \\
& +\ldots \ldots \\
& \left.+\operatorname{tr}_{\lambda}\left(\pi_{\lambda}\left(x_{k_{1}}\right) \pi_{\lambda}\left(x_{k_{2}}\right) \ldots \pi_{\lambda}\left(x_{k_{m}}\right)\right) \operatorname{tr}_{\mu}\left(\pi_{\mu}\left(x^{k_{1}}\right) \pi_{\mu}\left(x^{k_{2}}\right) \ldots \pi_{\mu}\left(x^{k_{m}}\right)\right)\right] .
\end{aligned}
$$

Now let us introduce a set of basis vectors $|\alpha\rangle$ for $V(\mu)$. Then we may write

$$
\begin{aligned}
\operatorname{tr}_{\lambda \otimes \mu}\left(A^{m}\right)=( & \left.-\frac{1}{2}\right)^{m} \sum_{\alpha, \mathbf{k}}\left[\operatorname{tr}_{\lambda}\left(\pi_{\lambda}\left(x^{k_{1}}\right) \pi_{\lambda}\left(x^{k_{2}}\right) \ldots \pi_{\lambda}\left(x^{k_{m}}\right)\right)<\alpha\left|\pi_{\mu}\left(x_{k_{1}}\right) \ldots \pi_{\mu}\left(x_{k_{m}}\right)\right| \alpha\right\rangle \\
& +\operatorname{tr}_{\lambda}\left(\pi_{\lambda}\left(x^{k_{1}}\right) \pi_{\lambda}\left(x^{k_{2}}\right) \ldots \pi_{\lambda}\left(x_{k_{m}}\right)\right)<\alpha\left|\pi_{\lambda}\left(x_{k_{1}}\right) \pi_{\mu}\left(x_{k_{\mathrm{g}}}\right) \ldots \pi_{\mu}\left(x^{k_{m}}\right)\right| \alpha> \\
& +\ldots \ldots \\
& \left.+\operatorname{tr}_{\lambda}\left(\pi_{\lambda}\left(x_{k_{1}}\right) \pi_{\lambda}\left(x_{k_{\mathrm{g}}}\right) \ldots \pi_{\lambda}\left(x_{k_{m}}\right)\right)<\alpha\left|\pi_{\lambda}\left(x^{k_{1}}\right) \pi_{\mu}\left(x^{k_{3}}\right) \ldots \pi_{\mu}\left(x^{k_{m}}\right)\right| \alpha>\right] \\
=( & \left.-\frac{1}{2}\right)^{m} \sum_{\alpha}<\alpha \mid \sum_{\mathbf{k}}\left(\operatorname{tr}_{\lambda}\left[\pi_{\lambda}\left(x^{k_{1}}\right) \pi_{\lambda}\left(x^{k_{2}}\right) \ldots \pi_{\lambda}\left(x^{k_{m}}\right)\right] \pi_{\mu}\left(x_{k_{1}}\right) \pi_{\mu}\left(x_{k_{2}}\right) \ldots\left(x_{k_{m}}\right)\right. \\
& +\operatorname{tr}_{\lambda}\left[\pi_{\lambda}\left(x^{k_{1}}\right) \pi_{\lambda}\left(x^{k_{2}}\right) \ldots \pi_{\lambda}\left(x_{k_{m}}\right)\right] \pi_{\mu}\left(x_{k_{1}}\right) \pi_{\mu}\left(x_{k_{2}}\right) \ldots \pi_{\mu}\left(x^{k_{m}}\right) \\
& +\ldots \ldots \\
& \left.+\operatorname{tr}_{\lambda}\left[\pi_{\lambda}\left(x_{k_{1}}\right) \pi_{\lambda}\left(x_{k_{2}}\right) \ldots \pi_{\lambda}\left(x_{k_{m}}\right) \pi_{\mu}\left(x^{k_{1}}\right) \pi_{\mu}\left(x^{k_{2}}\right) \ldots \pi_{\mu}\left(x^{k_{m}}\right)\right]\right) \mid \alpha>.
\end{aligned}
$$

Hence from equation (28) we obtain

$$
\operatorname{tr}_{\lambda \otimes \mu}\left(A^{m}\right)=\sum_{\alpha}\left\langle\alpha\left|I_{m}(\lambda)\right| \alpha\right\rangle .
$$

Since $I_{m}(\lambda)$ is an invariant of $L$ it follows that

$$
\operatorname{tr}_{\lambda \otimes \mu}\left(A^{m}\right)=I_{m}(\lambda) \operatorname{Dim} V(\mu)
$$




\section{References}

[1] G. E. Baird and L. C. Biedenharn, J. Math. Phys. 5 (1964), 1730-1747.

[2] A. J. Bracken and H. S. Green, J. Math. Phys. 12 (1971), 2099-2106.

[3] A. L. Carey, A. Cant and D. M. O'Brien, Ann. Inst. Henri Poincaré, 26A (1977), 405-429.

[4] S. A. Edwards, "A new approach to the eigenvalues of the Gel'fand invariants for the unitary, orthogonal and symplectic groups", Univ. of Adelaide: Dept. Math. Phys. Preprint.

[5] M. D. Gould, "Applications of the characteristic identity for $G L(n)$ ", Univ. of Adelaide: Dept. Math. Phys. Preprint.

[6] H. S. Green, J. Math. Phys. 12 (1971), 2106-2113.

[7] H. S. Green, J. Austral. Math. Soc. B 19 (1975), 129-139.

[8] H. S. Green, C. A. Hurst and Y. Ilamed, J. Math. Phys. 17 (1976), 1367-1382.

[9] K. C. Hannabuss, "Characteristic equations for semi-simple Lie groups", Math. Inst. Oxford Preprint (1972).

[10] J. E. Humphreys, Introduction to Lie algebras and representation theory (Springer-Verlag, New York-Heidelberg-Berlin, 1972).

[11] B. Kostant, J. Funct. Anal. 20 (1975), 257-285.

[12] B. Kostant, Trans. Amer. Math. 93 (1975), 528.

[13] C. O. Nwachuku and M. A. Rashid, ICTP Report IC/75/144 (1975).

[14] S. Okubo, J. Math. Phys. 16 (1975), 528.

[15] S. Okubo, Rochester Report UP-608 (1977).

[16] See, for example, Humphreys [10, p. 121].

Department of Mathematical Physics

University of Adelaide

Adelaide, South Australia, 5001 